\title{
Structure of the poleward wall of the trough and the inclination of the geomagnetic field above the EISCAT radar
}

\author{
D. G. Jones, I. K. Walker, L. Kersley \\ Department of Physics, The University of Wales, Aberystwyth, SY23 3BZ, UK \\ Received: 3 September 1996 / Revised: 27 January 1997 / Accepted: 5 February 1997
}

\begin{abstract}
A special high-resolution routine of the EISCAT radar has been used to investigate the structure and development of the poleward wall of a deep trough in electron density. The feature was tracked by the radar during a 7-hour period under very quiet geomagnetic conditions. The field-aligned nature of the structure enabled an estimate to be made of the inclination of the geomagnetic field above EISCAT that was in good agreement with the current model. Observations of narrow field-aligned enhancements in electron temperature demonstrated that the wall of this trough is a dynamic feature, reforming regularly as the electron density responds on a time scale of tens of minutes to energy input from soft-particle precipitation.
\end{abstract}

\section{Introduction}

Irregularities in the electron density at the poleward edge of a long-lived trough have been used to estimate the orientation of the geomagnetic field in the ionospheric F-layer. The geomagnetic field plays a key role in the dynamics of the plasma in the ionised atmosphere. Use is made in the interpretation of many experimental observations of the magnitude and direction of the geomagnetic field given by successive generations of the International Geomagnetic Reference Field Model. However, few measurements can be found in the literature of the geomagnetic field in space being determined from ground-based observations. One such measurement was that reported by Woodman (1971), where the aspect sensitivity of radar backscatter from field-aligned irregularities in the equatorial ionosphere above Jicamarca was used to estimate the geomagnetic field orientation. A discrepancy of about $1^{\circ}$ was found between the Jicamarca measurements and the then current field models which were based on satellite data.

The main trough is a well-established feature of the sub-auroral ionosphere. Comprehensive summaries of both observational and theoretical studies of the trough have been given by Moffett and Quegan (1983) and Rodger et al. (1992). Early experimental work of particular interest to the current investigation was reported by Taylor (1973). He described observations made using an incoherent scatter radar at Malvern in the UK, in which, during disturbed geomagnetic conditions the poleward wall of the trough moved over the radar. He estimated that this structure was tilted along the direction of the local geomagnetic field to within $\pm 4^{\circ}$. More recently, Collis and Häggström (1988) used some $450 \mathrm{~h}$ of common programme observations of the EISCAT radar to study the location of the trough in relationship to plasma convection and auroral precipitation. A key point was that the poleward edge of the trough was at the equatorial limit of auroral electron precipitation. Earlier work by Rino et al. (1983) had identified a boundary blob of enhanced plasma densities poleward of the trough, linked to latitudinally localised precipitation of lowenergy electrons. However, modelling work by Robinson et al. (1985) indicated that the boundary blob may result from a reconfiguration of structures produced well upstream in the convective flow.

The present work addresses the field-aligned nature of the poleward wall of the trough and its origins. It concerns study of a very steep meridional gradient in electron density that formed the poleward edge of the trough, observed over a 7-hour period during very quiet geomagnetic conditions. The field-aligned nature of the plasma structuring has been used to estimate the inclination of the geomagnetic field in the ionospheric F-region. In addition, the plasma diagnostics provided by the EISCAT radar give evidence for the mechanism responsible for the gradient. 


\section{The BLOB experiment}

The EISCAT special program SP-UK-BLOB was designed to study irregularities in the ionospheric plasma on scales of tens of kilometres transverse to the geomagnetic field. A full description of the experiment has been discussed by Pryse et al. (1996), so that only a brief summary is given here.

The BLOB special program makes use of the EISCAT UHF radar, which comprises a transmitter at Tromsø in Norway and receivers at Tromsø, Kiruna in Sweden and Sodankylä in Finland. Observations can be made of electron density, electron and ion temperatures and ion drift. At the outset of a BLOB run the radar makes a long search scan in the meridian from north to south. This search is of $30 \mathrm{~min}$ duration and covers a latitude range of some $7^{\circ}$ about Troms $\varnothing$ at an altitude of $325 \mathrm{~km}$. During scanning, range-corrected power profiles are plotted at Tromsø in real time on a colourcoded latitude versus height fan plot. The plot enables the experimenter to identify regions of electron density structuring. Once a feature has been located, the latitudinal extent of the scanning can be restricted so that only the region encompassing the structure of interest is viewed. The resulting short scans may be either southward or northward and their limited latitudinal extent means that a developing plasma structure can be observed with a much reduced cycle time. Further alteration of the latitude range during observations allows the experimenter to follow a feature if it is moving in latitude. Once the structure has decayed or convected out of the radar field of view, the scanning is reverted to the search mode of the long scan. A key feature of the program is that the radar has a dwell time of $10 \mathrm{~s}$ at each position and moves $0.05^{\circ}$ latitude at $325 \mathrm{~km}$, equivalent to about $6 \mathrm{~km}$ at F-region heights, between adjacent pointing directions. Thus irregularities in the plasma on scales of tens of kilometres can be identified, bearing in mind that the theoretical beamwidth of the radar is some 3 to $4 \mathrm{~km}$ at F-layer altitude.

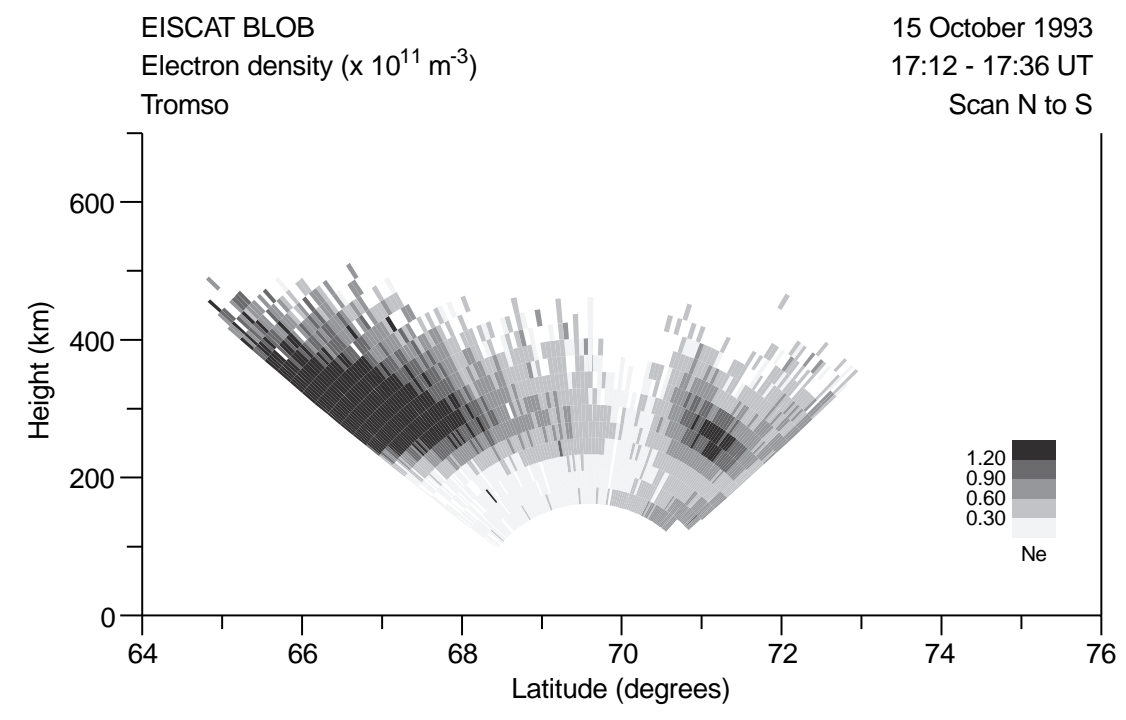

Fig. 1. Electron densities measured by the EISCAT radar during an initial scan of the SPUK-BLOB program between 1712 and 1736 UT on 15 October 1993
Pryse et al. (1996) reported results from an earlier run of the BLOB program where three different types of field-aligned irregularities were observed during a twohour period. One was a long-lived boundary blob of cold plasma. The second irregularity type was a narrow density enhancement associated initially with soft-particle precipitation. The third type was a narrow trough in electron density, associated with high ion temperatures that were caused by ion-frictional heating in fast convective flow. Further work indicated that both the boundary blob and the narrow density enhancement were convecting with the background plasma.

\section{Observations}

The observations used for the current study were made during a 7-h run of the BLOB special programme that took place on the evening of the 15 October 1993. Magnetic conditions were very quiet throughout the period of the run, with $K_{p}=0+$.

A fan plot obtained from the initial search scan of the radar is shown in Fig. 1, where electron density is presented as a function of height and latitude. A trough can be seen above the radar with a minimum in electron density at around $70^{\circ} \mathrm{N}$. A steep horizontal gradient in electron density forms the poleward wall, with a shallower gradient on the equatorward side. The steep gradient of the poleward wall was the focus of the present study. It proved possible to track this feature using the radar throughout the entire 7 -hour run of the experiment.

Figure 2 shows a series of electron density plots for different times throughout the progression of the run. It can be seen that the trough wall moved southwards with time so that the latitudinal range chosen for the different scans varied accordingly. For the scan commencing at 1829 UT (Fig. 2a), the latitudinal extent had been restricted to encompass the poleward wall of the trough, which can be seen clearly above $69.5^{\circ} \mathrm{N}$. At $300 \mathrm{~km}$ altitude, a fourfold increase in density occurred within a
15 October 1993

12 - 17:36 UT

Scan $\mathrm{N}$ to $\mathrm{S}$ 

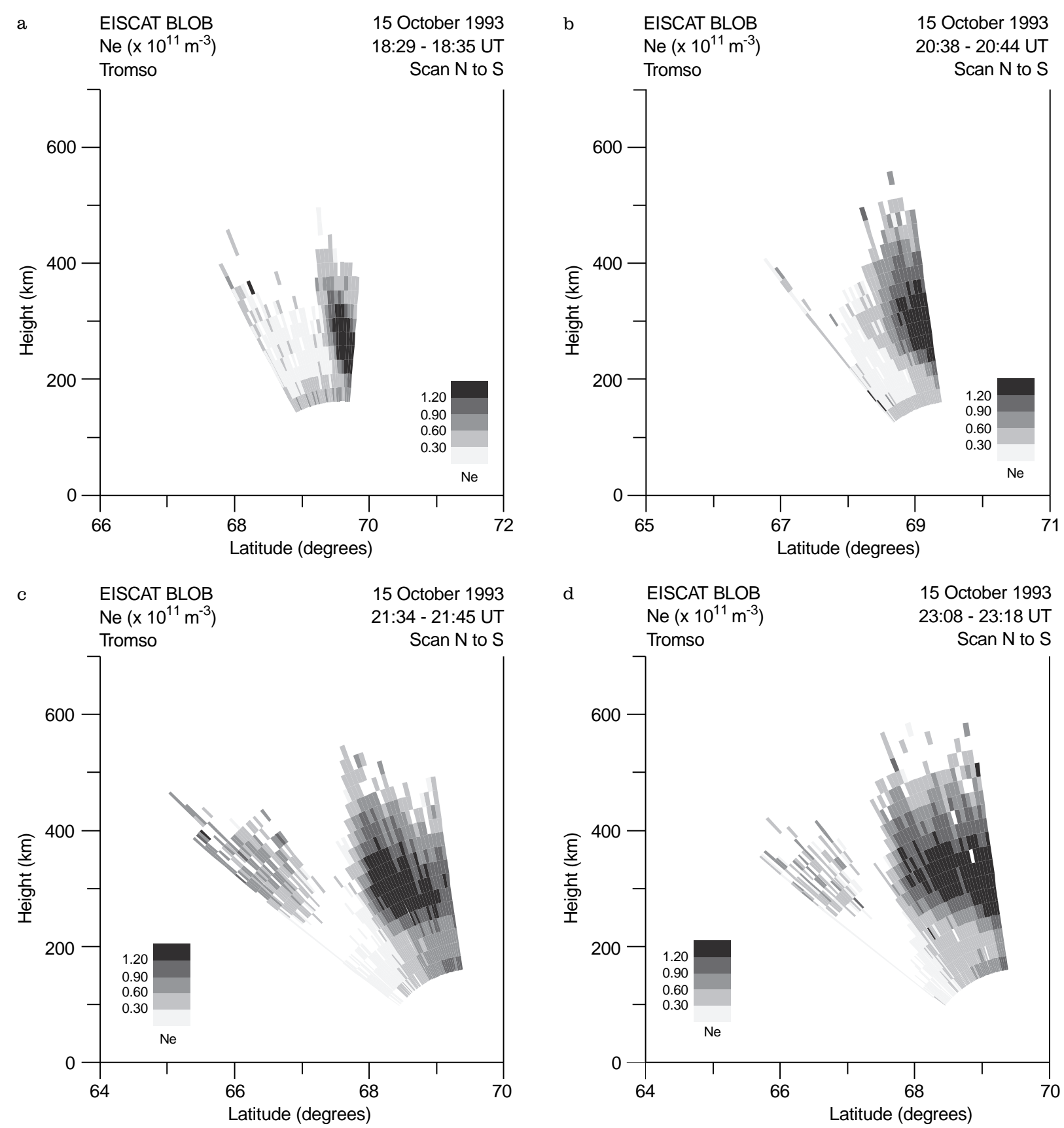

Fig. 2a-d. Electron densities from selected short scans of the EISCAT BLOB routine on the evening of 15 October 1993

quarter of a degree of latitude, equivalent to a horizontal distance of about $30 \mathrm{~km}$. It should be noted that areas where no shading is apparent within the fan plot represent gates where electron densities were so low that no useful signal was returned to the radar. Comparison of Fig. $2 \mathrm{a}$ with $2 \mathrm{~b}$ reveals that the trough has moved south by more than $0.5^{\circ}$ during the intervening $2-\mathrm{h}$ period. It is also apparent that the trough wall no longer exhibits such a steep gradient. The final two plots, Fig. 2c, d illustrate the electron densities for scans commencing at 2134 UT and 2308 UT respectively. The trough wall is again in evidence and it has progressed southwards to about $68^{\circ} \mathrm{N}$.

\section{Field alignment}

The complete sequence of plots showed that the poleward wall of the trough was consistently tilted in the direction of the local geomagnetic field. The observations were used to estimate the inclination of the field in the auroral ionosphere for comparison with that predicted by the IGRF90 model. However, it was first necessary to devise a consistent method for identifying the edge of the trough wall and to consider the effect of convective motion of the plasma during the scan of the radar. A number of selection criteria were established as follows. The poleward wall of the trough 
can be seen in Fig. 3, where the electron density is plotted as a function of latitude for the scan of the radar commencing at 1836 UT. The four curves correspond to interpolations of the measurements at heights ranging from $240 \mathrm{~km}$ to $300 \mathrm{~km}$ in $20 \mathrm{~km}$ intervals. It can be noted that this range spans the height of the layer peak, that in this instance was about $260 \mathrm{~km}$. However, the structures studied here show considerable uniformity of density along the field direction, unlike the normal profile of a solar-produced ionospheric F-layer. Attention has been concentrated in the present study on cases where the maximum density found in the boundary blob varied by not more than $10 \%$ over the chosen $60 \mathrm{~km}$ height range spanning the layer peak. In this way the effect of the background vertical profile has been minimised in the estimation of the orientation of the field direction. In the case shown in Fig. 3 the trough wall is clearly defined at each of the chosen heights. However, for some other scans, like the results shown in Fig. 4, there is evidence for further structuring within the gradient making assessment of the inclination more difficult. Figure 5 shows the electron density versus latitude for eight consecutive scans at a fixed height of $280 \mathrm{~km}$. It can be seen that for the first four scans the position of the trough wall is reasonably consistent, varying by less than $0.1^{\circ}$ latitude in the 27 min covered, regardless of the electron density level selected within the range of 0.3 to $0.9 \times 10^{11} \mathrm{~m}^{-3}$. However, in the transition to the subsequent four scans shown here, a significant shift in latitude is observed, with an implied average southwards velocity of some $40 \mathrm{~ms}^{-1}$. For this reason, the estimates of the geomagnetic field inclination were restricted to cases where comparison between adjacent observations showed that the bulk latitudinal motion of the plasma in the vicinity of the trough was less than about $10 \mathrm{~ms}^{-1}$. A further requirement was to choose examples where the trough wall was positioned

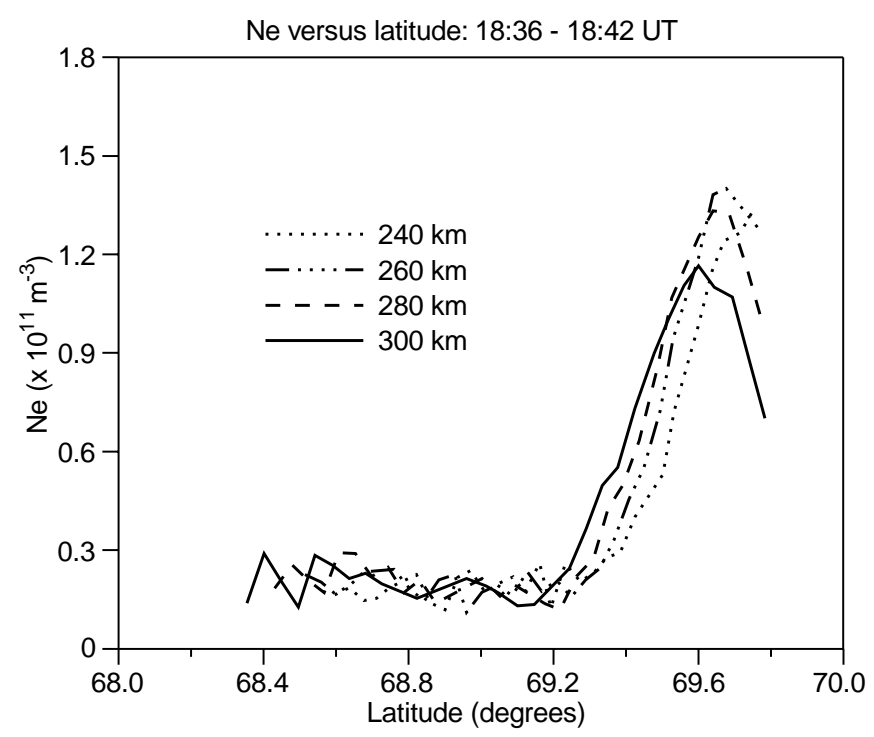

Fig. 3. Electron densities as a function of latitude for four selected heights measured by the radar during the scan commencing at 1836 UT

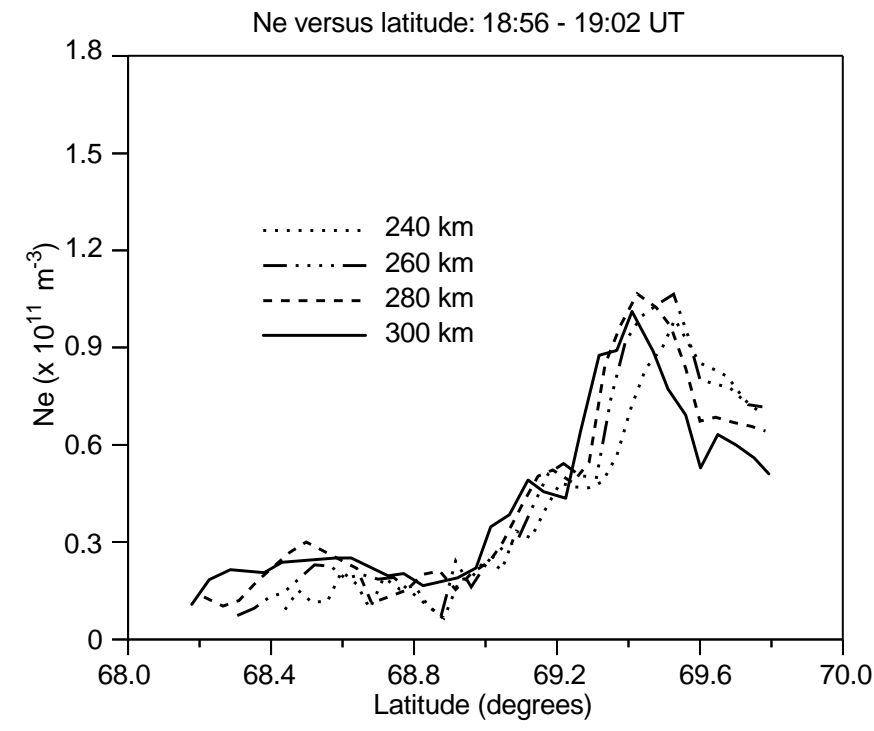

Fig. 4. Electron densities as a function of latitude for four selected heights measured by the radar during the scan commencing at 1856 UT

roughly on the same L-shell. According to IGRF90, the inclination of the field changes by about $0.1^{\circ}$ for every $0.2^{\circ}$ latitude in this region. It was found that observations from 5 scans complied with all of these criteria, the position of the wall being slightly equatorward of the radar at a latitude $69.4^{\circ} \pm 0.1^{\circ}$ in these examples.

A number of selected thresholds of electron density were chosen to correspond to the levels found on the wall gradient and the latitude of these determined at each of the four heights over the $60 \mathrm{~km}$ range. The results were differenced with respect to both a base latitude and a lower reference altitude and plotted in terms of relative latitude against height above the

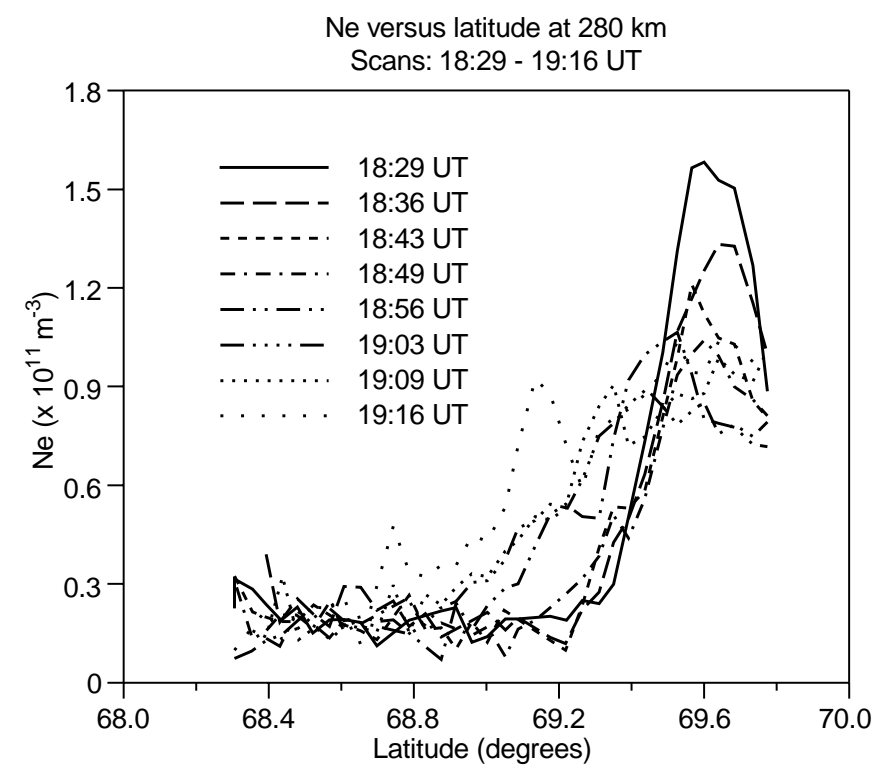

Fig. 5. Electron densities at $280 \mathrm{~km}$ altitude as a function of latitude measured during eight successive scans of the radar, each identified by the start time 
chosen base of $240 \mathrm{~km}$. In total, 21 sets of points were determined for the chosen threshold densities from the five examples conforming to the selection criteria. A plot showing the means and standard deviations derived from these points is given in Fig. 6. The best-fit line through all 21 data sets has been used to estimate the orientation of the geomagnetic field, over the $60 \mathrm{~km}$ height range near the F-layer peak at the location marginally south of the EISCAT Tromsø station. This was found to be $77.4^{\circ} \pm 0.7^{\circ}$. This value can be compared favourably with that obtained using the IGRF90 model of $77.7^{\circ} \pm 0.1^{\circ}$, over the height range 275 to $500 \mathrm{~km}$. The error margin quoted in this latter case is an estimate of the maximum error in the inclination, taken from Langel (1992). It can be noted that the EISCAT measurements were obtained from radar scans in the geographic meridian. However, the declination of the geomagnetic field at $300 \mathrm{~km}$ above EISCAT is only a few degrees so that the change in the measured inclination when mapped into the geomagnetic meridian is much less than the experimental error quoted. It should also be noted that the measurements were made during an extended period of very quiet geomagnetic conditions so that the possible influences of disturbed activity on the orientation of the field were likely to be minimal.

\section{Physical mechanisms}

The strict field-alignment of the plasma structuring at the poleward edge of the trough provides insight into its origins, while the diagnostic capability of the EISCAT radar yields further evidence on the physical mechanisms responsible. Analysis of the incoherent scatter measurements gives values of electron and ion temperatures to the same high spatial resolution as the electron densities. While the ion temperatures in the vicinity of the trough wall show little evidence of defined structure, by contrast the electron temperatures are characterised

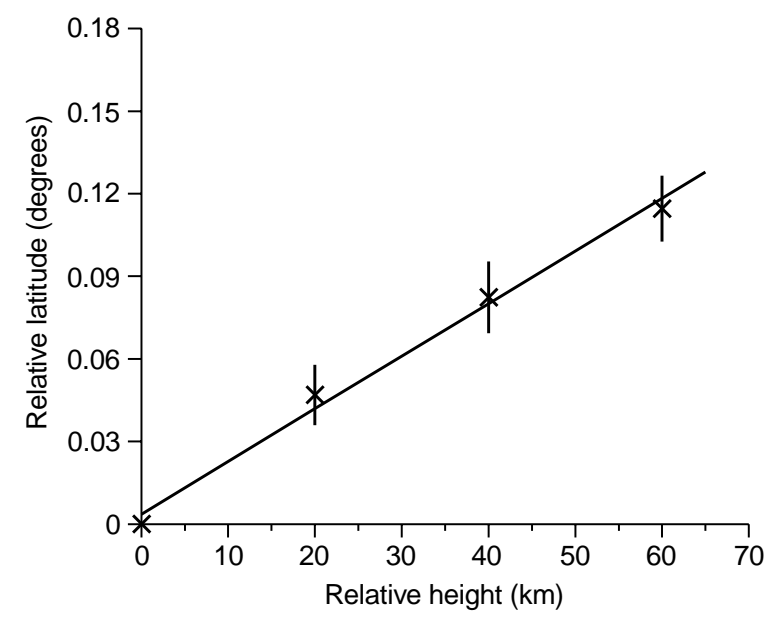

Fig. 6. Mean latitude of the poleward wall of the trough as a function of height, plotted with respect to that at a height of $240 \mathrm{~km}$. The error bars indicate plus and minus one standard deviation for the 21 sets of observations used in the study by field-aligned enhancements on a fine scale throughout much of the period of interest. Figure 7 shows a plot of the electron temperature measured during the radar scan between 1916 and 1922 UT that has features typical of many of the records throughout the run. The temperatures are low $(<900 \mathrm{~K})$ in the region of the density trough minimum in the south of the region scanned, but in the vicinity of the poleward wall there is a sharp onset to significantly higher temperatures and a narrow fieldaligned enhancement where the electron temperature exceeds $1700 \mathrm{~K}$. This structure extends throughout the F-region and is indicative of rapid heating of the electron gas by the precipitation of soft electrons down the field lines. The intense heating is very restricted in latitude covering only two or three radar pointing directions at any height, that is only some $20 \mathrm{~km}$ in width transverse to the field. There is also evidence for another narrow field-aligned temperature enhancement above $300 \mathrm{~km}$ at the northern extremity of the region scanned. It can be noted that the elevated electron temperatures do not appear in the plot to extend down into the lowest part of the F-layer, well below $200 \mathrm{~km}$. However, the analysis of the spectral returns from the radar was carried out using a standard model with a transition from an atomic to molecular ion mass in this region. In consequence, the temperatures plotted for the lowest range gates must be treated with some caution. In addition, since the BLOB routine was designed to study structures at F-layer heights, there is no information in Figure 7 of conditions in the underlying E-layer. It is thus not possible to say in this case whether the electron heating resulted from localised precipitation of soft electrons into the F-layer or was formed by the soft tail of a harder precipitation event down into the auroral Elayer.

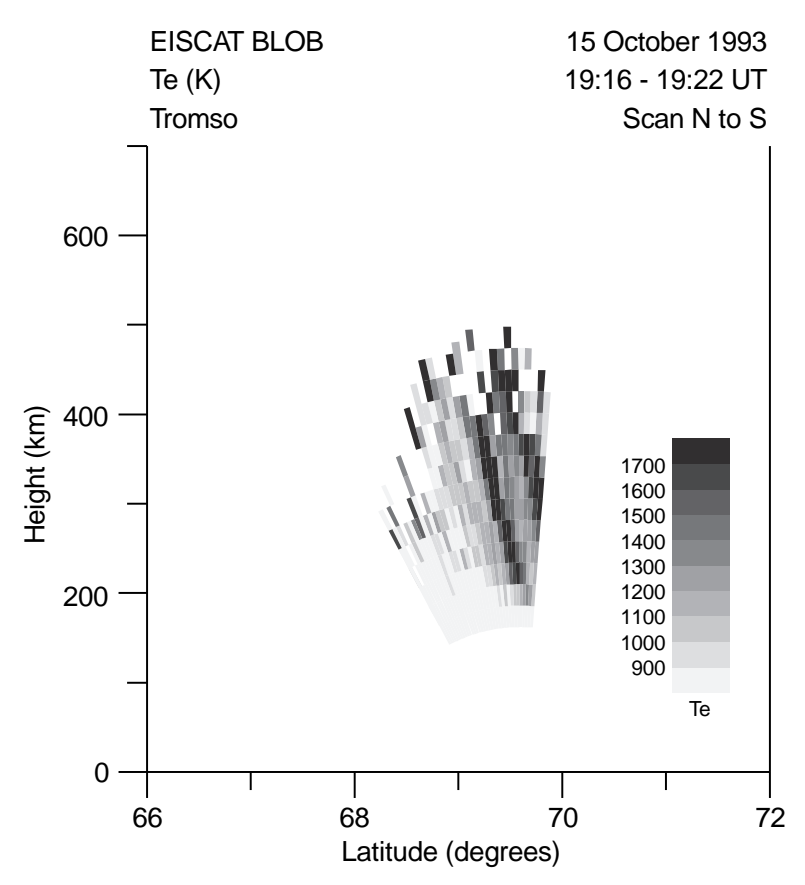

Fig. 7. Electron temperatures as a function of latitude and height measured by the radar during the scan between 1916 and 1922 UT 
The plot of electron density corresponding to Fig. 7 does not show a one-to-one relationship in field-aligned structuring with that seen in the electron temperature. However, the modelling work of Roble and Rees (1977) demonstrated that the response time of the electron density in the F-region to energy input from soft particles would be on a time scale of tens of minutes, in contrast to the more nearly instantaneous rise in the electron temperatures. It is thus to be expected that the electron densities and temperatures are not immediately related. However, the latitudinally narrow field-aligned enhancements in electron temperature are characteristic of conditions poleward of the trough minimum during the entire period of the observations. Almost every scan of the radar shows evidence for such a region of softparticle precipitation, sometimes more diffuse than the example shown here, sometimes persisting at the same location for several scans, but on other occasions appearing as a transient feature. The field-aligned nature of the electron density gradient at the poleward edge of the trough is thus a direct consequence of its origins, as a response to energy input from soft-particle precipitation with a sharp equatorwards edge.

\section{Discussion and conclusions}

The steep gradient in electron density that forms the poleward wall of the main trough has been shown to be accurately aligned with the geomagnetic field, the estimate of the inclination of the field being made over a height range of about $60 \mathrm{~km}$. The measured value of the orientation of the field of $77.4^{\circ} \pm 0.7^{\circ}$ to the horizontal is in excellent agreement with the standard model computation. With a $K_{p}$ value of $0+$ throughout the period of the observations, the geomagnetic conditions were quiet, so that possible changes in the orientation due to the influence of magnetic activity were likely to be minimised.

The results demonstrate clearly that the poleward edge of the trough in this case is produced by the effects of the precipitation of soft-electrons into the ionospheric F-layer. This energy input is in the form of transient bursts down field lines, confined to a narrow band, often less than $20 \mathrm{~km}$ in latitudinal extent. The regions of electron heating are apparently for the most part short-lived, only occasionally being found at the same location on successive scans of the radar separated typically by some 6 to $10 \mathrm{~min}$. However, the equatorwards edge of the region of soft-particle precipitation was generally well-defined and it was this that determined the position of the poleward wall of the trough. Direct one-to-one correspondence between the energy input signified by regions of elevated temperature and the consequent enhancement in electron density is not possible for two reasons. The modelling work of Roble and Rees (1977) demonstrated that at F-layer altitudes, the response of the electron density to energy input from soft-particle precipitation would be delayed by some tens of minutes, though the electron temperature would show a nearly instantaneous rise. Furthermore, during this delay the plasma may have convected out of the radar beam. The plasma velocities measured from the tri-static capability of the EISCAT radar show that the observations encompassed the time of the flow reversal of the Harang discontinuity. However, in general, the zonal component in the convection is dominant so that it is often unlikely that the same region of plasma was sampled in successive scans of the radar. Exact correspondence between elevated electron temperatures and subsequent enhanced electron densities is thus not to be expected in the observations. However, careful examination of the results does provide evidence of later density increases in the vicinity of field lines showing high electron temperatures in earlier scans. Coupled with instances of elevated electron temperatures at similar latitudes in more than one adjacent scan, this provides evidence that the regions of soft-particle precipitation are extended longitudinally. However, the errors in the zonal plasma velocities estimated by the radar are large due to the short integration time, so that the exact longitudinal extent cannot be estimated with any reliability.

It can be noted that the inclination of the geomagnetic field was also estimated from the structures of increased electron temperature, using selection criteria similar to those already described. Indeed, this approach, though not based on a physical structure like the density gradient, may be an improvement on the previous method as the increase in electron temperature is essentially instantaneous following the input of energy from the precipitating particles, whereas electron densities at F-region altitudes increase over a period of tens of minutes, by which time convection may have distorted the structure. The position of the feature was defined by the peak electron temperature at each $20 \mathrm{~km}$ height interval. In this case the height range was not affected by the background profile, so it was possible to use measurements over a range from $200 \mathrm{~km}$ to $400 \mathrm{~km}$ altitude. A drift velocity of less than $10 \mathrm{~ms}^{-1}$ and a similar latitudinal position for each example were again used as selection criteria. Six examples were found that yielded an estimate for the inclination of the geomagnetic field of $77.7^{\circ} \pm 0.7^{\circ}$, again comparing well with the model value of $77.8^{\circ} \pm 0.1^{\circ}$ at this latitude.

The formation of the poleward wall of the F-layer trough is thus characterised by transient discrete regions of field-aligned soft-particle precipitation, of scale often less than $20 \mathrm{~km}$ transverse to the field but more extended longitudinally. The wall develops in an equatorwards direction as a new burst of precipitation, at a slightly lower latitude than the previously well-defined limit, is followed by a build-up of electron density some tens of minutes later. An example of this build-up can be seen above $69.2^{\circ} \mathrm{N}$ in Fig. 4 as the wall begins to reform at a slightly lower latitude.

It can be noted that the latitudinal range over which these precipitation events occur tends to be very restricted so that the wall gradient is often surmounted by a boundary blob and a subsequent decrease in density to the north. The boundary blob in this instance is a long-lived, though dynamic feature, constantly being 
reformed as a result of latitudinally restricted, transient bursts of soft-particle precipitation, though it must be noted that the maximum densities of $\sim 10^{11} \mathrm{~m}^{-3}$ are generally low in this case. The classic example of a boundary blob, observed by Rino et al. (1983) and subsequently modelled by Robinson et al. (1985), was of cold plasma being reconfigured into a stable structure after convection across the polar cap. This is certainly not the case in the situation discussed here where the plasma blob at the polewards edge of the trough, although present for many hours, is a dynamic feature constantly undergoing a process of reformation in response to localised in situ energy input.

Acknowledgements. The co-operation of the director and staff of the EISCAT Scientific Association is gratefully acknowledged. Thanks are also due to the EISCAT group at the Rutherford Appleton Laboratory and in particular for the contributions of Dr Alan Aylward and Mr Steve Crothers in the development and implementation of the SP-UK-BLOB routines. Values for the inclination of the geomagnetic field above Troms $\varnothing$ were computed using the 6th Generation International Geomagnetic Reference Field Model 1990, (IGRF90), provided by the British Geological Survey's Geomagnetism Group Information Service. IKW is a PDRA and DGJ a postgraduate student, both supported by PPARC.

Topical Editor D. Alcayde' thanks J. Sojka and M. Förster for their help in evaluating this paper.

\section{References}

Collis P. N., and I. Häggström, Plasma convection and auroral processes associated with the main ionospheric trough at high latitudes, J. Atmos. Terr. Phys., 50, 339-404, 1988.

Langel R.A., International Geomagnetic Reference Field: the sixth generation, J. Geomag. Geoelectr., 44, 679-707, 1992.

Moffett R. J., and S. Quegan, The mid-latitude trough in the electron concentration of the ionospheric F-layer: a review of observations and modelling, J. Atmos. Terr. Phys., 45, 315-343, 1983.

Pryse S. E., L. Kersley, and I. K. Walker, Blobs and irregularities in the auroral ionosphere, J. Atmos. Terr. Phys., 58, 205-215, 1996.

Rino C. L., R. C. Livingston, R. T. Tsunoda, R. M. Robinson, J. F. Vickrey, C. Senior, M. D. Cousins, J. Owen, and J. A. Klobuchar, Recent studies of the structure and morphology of auroral zone F region irregularities, Radio Sci., 18, 1167-1180, 1983.

Robinson R. M., R. T. Tsunoda, J. F. Vickrey, and L. Guerin, Sources of $\mathrm{F}$ region ionization enhancements in the nighttime auroral zone, J. Geophys. Res., 90, 7533-7546, 1985.

Roble R. G., and M. H. Rees, Time-dependent studies of the aurora: effects of particle precipitation on the dynamic morphology of ionospheric and atmospheric properties, Planet. Space Sci., 25, 991-1010, 1977.

Rodger A. S., R. J. Moffett, and S. Quegan, Review paper: the role of ion drift in the formation of ionisation troughs in the midand high-latitude ionosphere - a review, J. Atmos. Terr. Phys., 54, 1-30, 1992.

Taylor G. N., Structure at the poleward edge of a mid-latitude Fregion trough, J. Atmos. Terr. Phys., 35, 647-656, 1973.

Woodman R. F., Inclination of the geomagnetic field measured by an incoherent scatter technique, J. Geophys. Res., 76, 178-184, 1971. 